\title{
Chapter 6 \\ Summary
}

The present study shows that the graded dielectric inhomogeneous radome exhibits better EM performance characteristics than monolithic half-wave and sandwich radome wall configurations (with either CTR or VTR designs) and provides a bandwidth of $500 \mathrm{MHz}$ from 9.75 to $10.25 \mathrm{GHz}$ with center frequency at $10 \mathrm{GHz}$. The proposed design gives superior performance for the entire forward hemisphere in terms of co-pol $(>-0.5 \mathrm{~dB})$, cross-pol $(<-30 \mathrm{~dB})$, boresight error $(<3 \mathrm{mrad})$ and also shows minimal pattern degradation. The excellent EM performance characteristics together with fabrication easiness make graded dielectric radome an exceptional design for both airborne and ground-based radome applications. 